\title{
Successful management of aorto-conduit fistula after esophagectomy: Tips and tricks to avoid catastrophe
}

\author{
Andrew F. Feczko, MD, ${ }^{\mathrm{a}}$ Monisha Sudarshan, MD, ${ }^{\mathrm{a}}$ Christopher J. Smolock, MD, ${ }^{\mathrm{b}}$ and
} Siva Raja, $\mathrm{MD}, \mathrm{PhD},{ }^{\mathrm{a}}$ Cleveland, Ohio

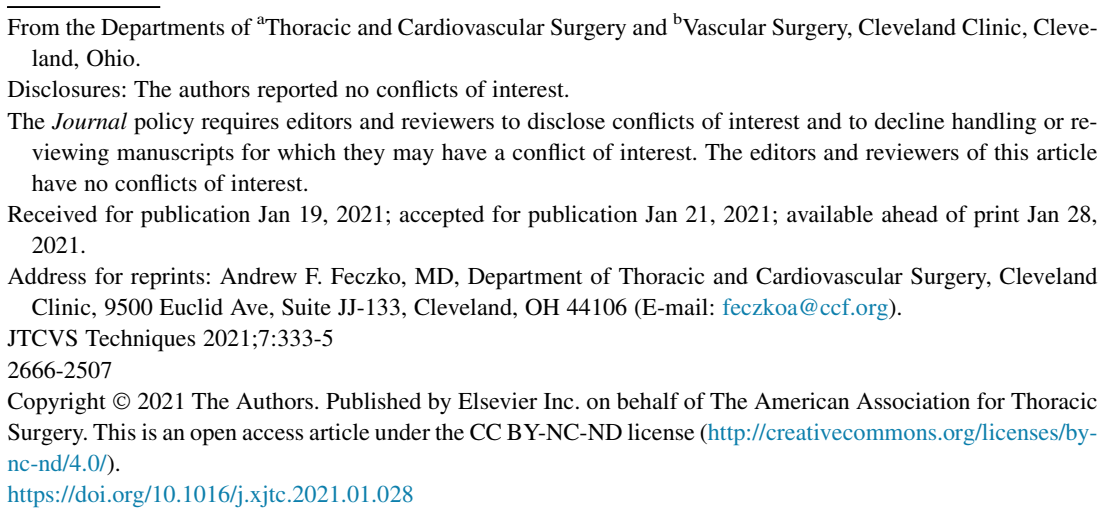

Aorto-conduit fistula (ACF) is a rare and morbid complication after esophagectomy. We present the case of a successful multidisciplinary rescue and repair of an ACF.

\section{CASE PRESENTATION}

A 76-year-old man presented to an outside hospital with massive hematemesis 9 months following an uncomplicated McKeown esophagectomy for ypT3 N1 adenocarcinoma. After initial endoscopic control of a known ulcer, he had recurrent hematemesis and was transferred to our institution in an emergency situation. Endoscopy demonstrated a large volume of clot and active bleeding without an identifiable source. Due to the failure of endoscopic control and presumed ulcer as a bleeding source, he was taken to interventional radiology for diagnosis and attempted embolization. An aortogram (Figure 1) ultimately revealed an ACF just proximal to the celiac and superior mesenteric arteries without a sufficient landing zone for endovascular stenting. As a temporizing measure, an occlusive aortic balloon was placed at the level of the fistula and the patient was taken urgently for operative exploration. A left thoracoabdominal approach was used, allowing excellent visualization of the entire conduit and aorta. Before dissecting the fistula, the aorta was controlled using supraceliac clamping and repositioning of the occlusive balloon below the fistula. A 2-mm aortic defect was identified adjacent to an area of inflammation on the conduit and was closed with a small bovine pericardial patch over the friable aortic tissue. The conduit was healthy and a $3-\mathrm{cm}$ defect was closed primarily and omentum interposed. Biopsy of this site was negative for malignancy. The patient returned to the operating room 3 days later for definitive closure and placement of additional vascularized tissue (thymus/pericardium) between the aorta and conduit. He ultimately recovered

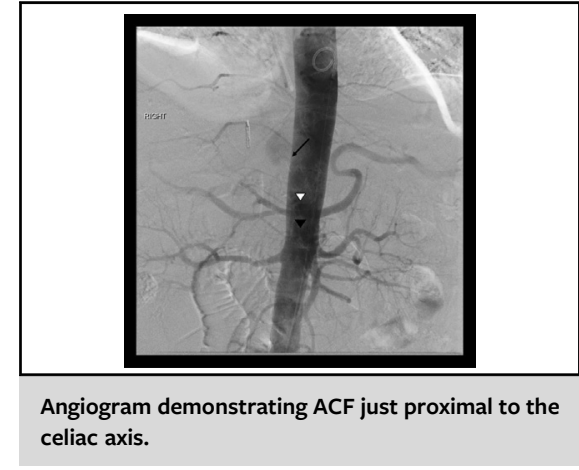

CENTRAL MESSAGE

Management of aorto-conduit fistula requires a multidisciplinary approach and emergency operative intervention with access to the thorax and abdomen.

See Commentaries on pages 336 and 338.

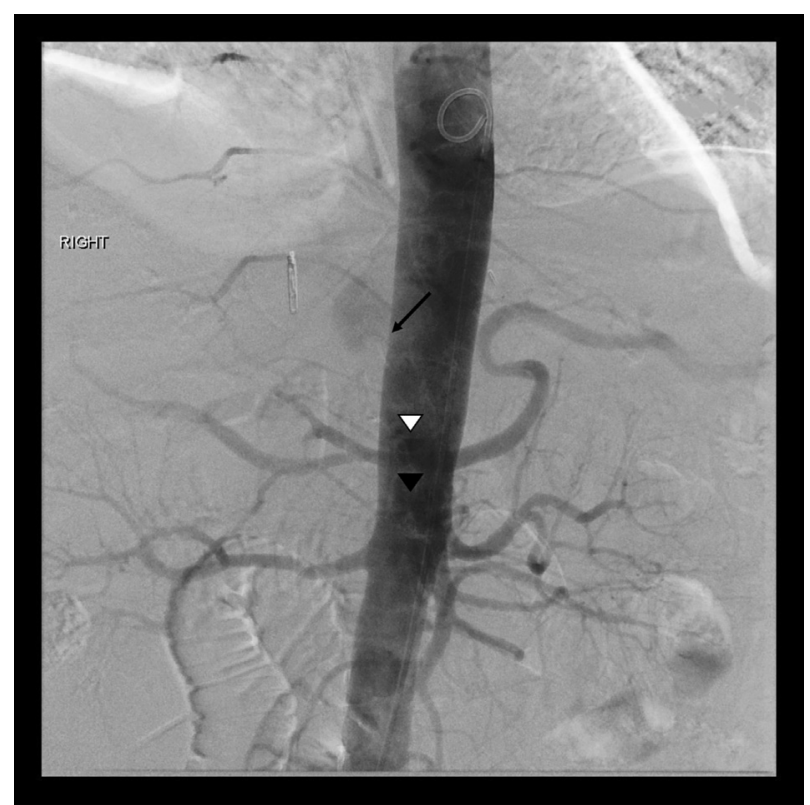

FIGURE 1. Aortogram demonstrating extravasation of contrast into gastric conduit via aorto-conduit fistula (ACF) (long arrow). The celiac (white arrowhead) and superior mesenteric artery (black arrowhead) are identified in close proximity to the ACF. 


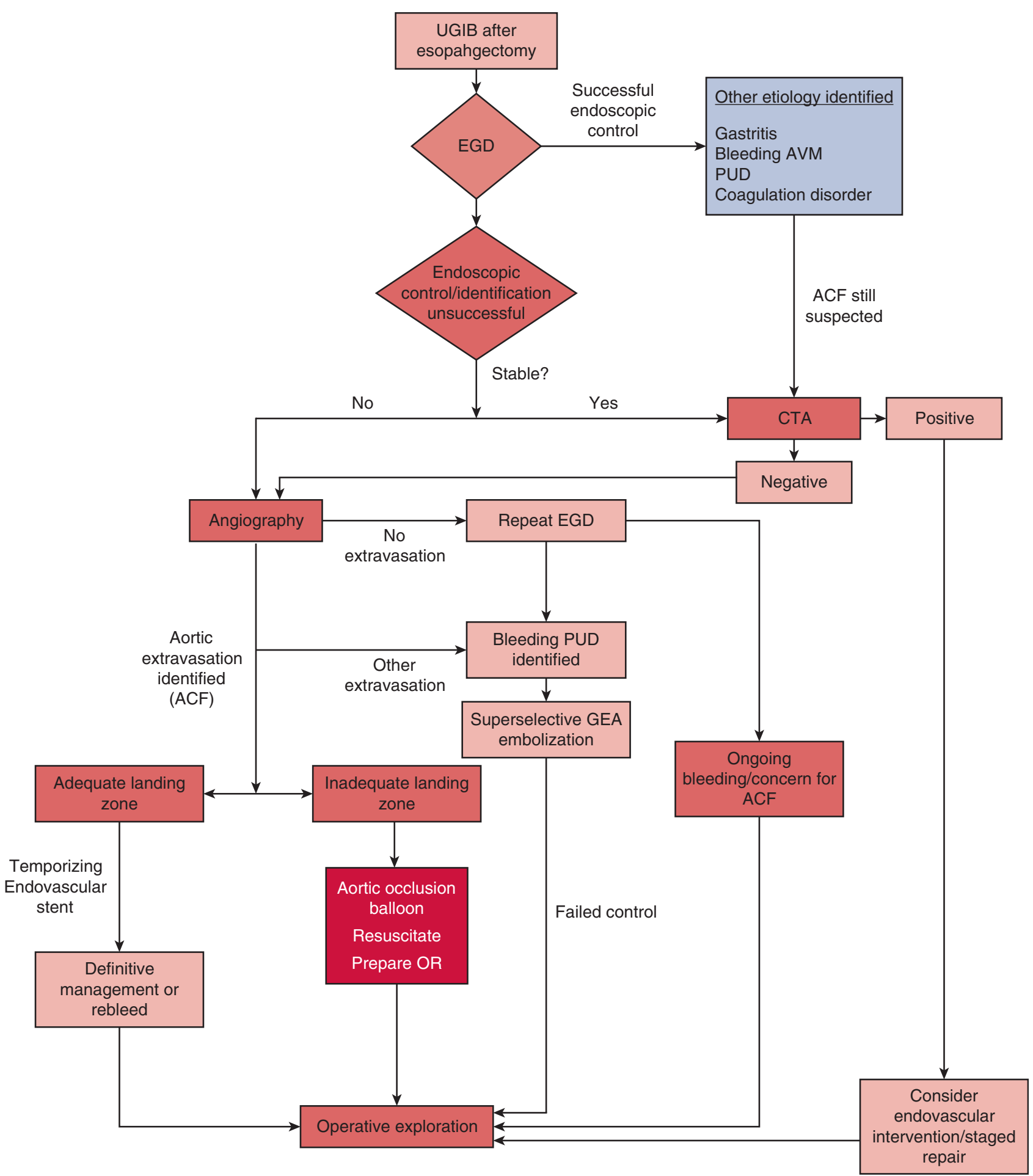

FIGURE 2. Proposed algorithm for the emergent management of an aorto-conduit fistula $(A C F)$. Sequential investigation using endoscopy and angiography is essential when ACF is suspected. High index of suspicion and additional evaluation is important in the setting of upper gastrointestinal bleed (UGIB) following esophagectomy. EGD, Esophagogastroduodenoscopy; $A V M$, arteriovenous malformation; PUD, peptic ulcer disease; $C T A$, computed tomography angiography; GEA, gastroepiploic artery.

and was discharged home on postoperative day 22. Unfortunately, following this challenging period, he died as a result of complications of metastatic disease. Permission to present case details was obtained. 


\section{DISCUSSION}

ACF is a rare but fatal complication following esophagectomy, representing a diagnostic and management challenge. Etiologies include chronic inflammation following a leak, ${ }^{1,2}$ surgical techniques (stripping of aortic adventitia), and persistent ulcer disease (as in this case). Both early (within 2-3 weeks) and late ( $>10$ years) ACFs have been described, with survival rates of $0 \%$ to $0.4 \% .^{1,3}$

Early diagnosis, rapid control, and definitive repair of the aorta and conduit are cornerstones of successful management (Figure 2). A herald bleed demands urgent investigation because massive hemorrhage can occur within minutes to days, but esophagogastroduodenoscopy is an appropriate first step to exclude more common causes of upper gastrointestinal hemorrhage. ${ }^{1}$ With aortic bleeding, visualization or endoscopic control of the fistula is impossible. Moving to the operating room at this stage is tempting when ACF is suspected, but this can create its own challenges when the level of the ACF is unknown. Moreover, proceeding to exploration without vascular control allows ongoing bleeding and conduit distension. Gaining a degree of temporary control, and better defining the ACF when the diagnosis is made can introduce a measure of control into an unstable situation.

Angiography can confirm an ACF, define the anatomy, and prepare for endovascular interventions. Although covered endovascular stents have been applied to other aortoenteric fistulae to control bleeding, coverage of the celiac axis after a standard gastric conduit risks conduit necrosis, trading 1 catastrophe for another. Placement of endovascular stents in the setting of an $\mathrm{ACF}$ also requires suppressive antibiotic therapy, and reintervention is often necessary. ${ }^{4} \mathrm{As}$ in this patient's case, placement of an occlusive aortic balloon under angiographic guidance can buy time to resuscitate and reach an operating room. Repositioning of the aortic balloon to above or below the fistula can provide proximal or distal control intraoperatively, minimizing dissection and hemorrhage. The assistance of vascular surgery or interventional radiology at this stage can be invaluable.

Successful operative management of an ACF requires simultaneous access to the thoracic, abdominal, and retroperitoneal cavities. The broad exposure provided by a thoracoabdominal incision is particularly important when the fistula may be infradiaphragmatic, allowing supracelic proximal aortic control (distal provided by the aortic occlusive balloon) and exposure to both the gastric conduit and aortic fistula. Careful attention should be paid to basic reconstructive principles, repairing vascular structures in a contaminated field with biologic materials (bovine pericardium), and interposition of 1 or more wellvascularized tissue flaps (eg, omentum, thymus, or pericardium).

$\mathrm{ACF}$ is a rare and highly morbid complication of esophagectomy; successful rescue involves early recognition and expeditious management. Based on our experience with this patient, we propose a multidisciplinary algorithm for evaluation and management, emphasizing early control and direct operative intervention. Cohesive involvement of gastroenterology, interventional radiology, vascular surgery, and anesthesia is essential in managing these challenging patients.

\section{References}

1. Molina-Navarro C, Hosking SW, Hayward SJ, Flowerdew ADS. Gastroaortic fistula as an early complication of esophagectomy. Ann Thorac Surg. 2001;72 1783-8.

2. Strong S, Higgs S, Streets C, Titcomb D, Barham P, Blazeby J, et al. Aorto-condui fistula developing four years after esophagectomy. J Surg Case Rep. 2012;2012:8.

3. Chotai HS, Finch G, Thomas D, Libertiny G. Successful management of an aortogastric fistula occurring 15 years after oesophagectomy with covered aortic stent graft placement followed by open surgery. J Surg Case Rep. 2018;2018:rjy019.

4. Russell T-AT, Pinnamaraju P, Hamish M. The role of TEVAR in the management of a recurrent aorto-gastric fistula. J Surg Case Rep. 2018;2018:rjy014. 